\title{
Some risk factors and effective agents on cardiovascular diseases incidence: The cross-sectional study in three cities in Southwest of Iran
}

\author{
Research Article
}

\section{Fateme Toulideh ${ }^{1}$, Hadi Mashali ${ }^{2}$, Mostafa Madmoli ${ }^{3}$, Rezvan Rahmani ${ }^{*}$}

1. Department of Nursing, Masjed-Soleiman branch, Islamic Azad University, Masjed-Soleiman, Iran

2. Nursing student, Student Research Committee, Behbahan Faculty of Medical Sciences, Behbahan, Iran

3. Emergency Medical Technician, Gerash University of Medical Sciences, Gerash, Iran

\begin{abstract}
Introduction: Among the chronic diseases, cardiovascular disease is the most important and most common cause of death in many countries. The aim of this study was to determine some risk factors and effective agents on cardiovascular diseases incidence by studying the cases of 705 patients referred to the hospital of 22 Bahman in Masjed Soleyman that were residing in Masjed Soleyman, Lali and Andika. Materials and Methods: In this retrospective cross-sectional analytical descriptive study, 705 cases of patients with cardiovascular disease admitted to the Masjed Soleyman Hospital of 22 Bahman that lived in the Masjed Soleyman, Lali and Andika, which were studied for 3 years from 2016 to 2018. Data were then entered into SPSS software version 20 and analyzed by descriptive statistics, analytical tests and significant level of $\mathrm{P}<0.05$. Results: The study sample included 705 cardiovascular disease patients with a mean age of $63.84 \pm 14.83 .12 .6 \%$ of patients had a history of smoking, 98.7 percent of patients used the drug, $2.3 \%$ had a history of vision problems, $12.8 \%$ had a history of kidney problems, $6.4 \%$ had a history of digestive problems, $16.9 \%$ had congestive heart failure, $25.7 \% \mathrm{MI}, 24.1 \%$ HTN, 25.7\% UA, 5\% bradycardia and 2.7\% had a tachycardia. Cardiovascular disease is also found in people with a poorer economic status, lower education, people with family history of heart disease, smoking (especially unstable angina and myocardial infarction), positive troponin (especially in unstable angina and myocardial infarction), had a history of pulmonary and renal problems there were statistically significant more than others (P $>$ 0.05). Conclusion: The results of this study showed that cardiovascular disease is also found in people with a poorer economic status, lower education, people with family history of heart disease, smoking (especially unstable angina and myocardial infarction), positive troponin (especially in unstable angina and myocardial infarction), had a history of pulmonary and renal problems there were statistically significant more than others. Therefore, it is necessary to take necessary educational measures to reduce some of these risk factors.
\end{abstract}

Keywords: Cardiovascular Disease, Risk Factors, Effective Factors, Congestive Heart Failure, MI.

\section{Introduction}

Among the chronic diseases, cardiovascular disease is the most important and most common cause of death in many countries (1-3) In all countries, the risk factors for cardiovascular disease are associated with a significant health burden (4). The risk factors for these diseases are hyperlipidemia (increased LDL, cholesterol and triglyceride levels), diabetes history, high blood pressure, obesity, low activity, smoking and psychological stress, and other factors including age, sex, ethnicity and a family history that can be controlled. Many of these risk factors start from childhood and adolescence, and are then habitually established in adolescent behaviors (5.6). At present, there has been a dramatic increase in the number of deaths from cardiovascular disease in the world, and is expected to reach 1.7 million deaths in 1999 to 1.11 million deaths in 2020 (7). In Iran, according to the

*Corresponding Author:

Rezvan Rahmani,

Department of Nursing,

Masjed-Soleiman branch,

Islamic Azad University,

Masjed-Soleiman, Iran.

Email: rezvanrahmani@gmail.com
WHO report, total deaths from cardiovascular disease are expected to reach $44.4 \%$ in 2030 (8). According to statistics released by the Ministry of Health and Medical Education, 303 cases of cardiovascular disease are reported in every 812 deaths in Iran (9). These diseases impose huge costs on the health systems of societies and while cardiovascular disease is one of the most preventable human diseases (10). Cardiovascular disease can also affect various aspects of physiological, physical, social, economic, mental, psychological and emotional life Patients will be affected (11). At its 2002 meeting, the World Health Organization (WHO) has emphasized the program of care for these risk factors in primary health services (12). Several studies have been conducted on the risk factors and factors affecting cardiovascular disease. Mousavinasab et al., In a study that investigated the factors affecting cardiovascular disease in people age 35 , cited that age, sex, fasting blood glucose, HDLcholesterol and triglycerides were associated with an increase in the severity of coronary artery bypass grafting (13). In their study, Mohtasham Amiri found that $13.4 \%$ had no cardiovascular risk factors, $6.49 \%$ had only one risk factor and $30.7 \%$ had two or more risk factors (14). The more people are exposed to more risk factors, the more likely they are to develop heart disease (15). By increasing the level of education, screening and controlling these factors, it can be prevented from 
developing cardiovascular disease or by delayed (16). Informing people about the role of risk factors in developing cardiovascular disease can be a way to combat these risk factors. The aim of this study was to determine Some risk factors and effective factors on cardiovascular diseases incidence by studying the cases of 705 patients referred to the hospital of 22 Bahman in Masjed Soleyman that were residing in Masjed Soleyman, Lali and Andika.

\section{Materials and Methods}

In this retrospective cross-sectional analytical descriptive study, 705 cases of patients with cardiovascular disease admitted to the Masjed Soleyman Hospital of 22 Bahman that lived in the Masjed Soleyman, Lali and Andika, which were studied for 3 years from 2016 to 2018 . The records, which were in the medical records of this hospital, were examined by two investigators. The patients were diagnosed with any cardiovascular disease and a history of the disease, aged over 30 years old and referred to the 22 Bahman Hospital of Masjed Soleyman from 2016-2018. After obtaining the necessary permissions from the Islamic Azad University of Masjed Soleyman, this license was referred to the Masjed Soleyman Healthcare Network. Then, the health network issued the necessary permission to the 22 Bahman Hospital and their files were used for this study.

The data needed for the study was extracted from patients' records during the years in the field of medical records of the hospital. The inclusion criteria included all patients with medical diagnosis and history of cardiovascular disease and age over 30 years old and in each age group and sex, and the exit criteria included other records of patients that had a medical diagnosis other than cardiovascular disease, files older than 30 years of age, as well as incomplete cases.

In order to study the files and collect data, a written Letter of Introduction was received from Masjed Soleyman Health Network Then, records of patients referring to the hospital in the archives department were used. That the required information was collected through a researcher checklist from the records. The data of this study included demographic, laboratory and clinical data such as age, sex, marital status, ethnicity, occupation, economic status, education level, smoking, systolic and diastolic blood pressure, blood glucose, troponin, history of drug and Family and personal history of diseases such as cardiovascular disease, diabetes, ocular, gastrointestinal and pulmonary diseases were investigated.

Data was then entered into SPSS software version 20 and using descriptive statistics and analytical tests of Chi-square and Pearson's chi-squared, ANOVA and significance level of $\mathrm{P}<0.05$ were analyzed.

\section{Results}

The study sample included 705 cardiovascular disease patients with a mean age of $63.84 \pm 14.83$. Of these, $51.2 \%$ were male and the rest were female and $66.5 \%$ had under diploma education.

Of these, $26.2 \%$ had diabetes, $18.4 \%$ had a family history of diabetes, $86.8 \%$ had a history of heart disease and $50.8 \%$ had a family history of heart disease. Troponin testing was positive in $30.6 \%$ of cases. $12.6 \%$ of patients had a history of smoking, 98.7 percent of patients used the drug, $2.3 \%$ had a history of vision problems, $12.8 \%$ had a history of kidney problems, $6.4 \%$ had a history of digestive problems.

$16.9 \%$ had congestive heart failure, $25.7 \% \mathrm{MI}$, $24.1 \%$ HTN, $25.7 \%$ UA, 5\% bradycardia and $2.7 \%$ had a tachycardia.

The mean blood glucose level of these patients was $151.55 \pm 87.62$, the average systolic blood pressure was $144.56 \pm, 33.81$ and the mean diastolic blood pressure of these individuals was $83.94 \pm 32.13$.

The ANOVA results showed a significant relationship between age and type of heart disease ( $P$ $<0.0001$ ), so that the mean age in congestive heart failure is greater and less in tachycardia patients.

Chi-square test showed a significant relationship between gender and type of cardiovascular disease ( $P$ $<0.0001)$. This means that the prevalence of hypertension and unstable angina in women and the prevalence of congestive heart failure and myocardial infarction was higher in men. (Figure 1)

There was no significant relationship between ethnicity and marriage and type of cardiovascular disease $(\mathrm{P}<$. $(0.05$ However, there was a significant relationship between employment status and type of cardiovascular disease $(\mathrm{P}<0.0001)$, in this way, housewives had more cardiovascular disease, particularly hypertension and unstable angina, than the rest.

Cardiovascular disease is also found in people with a poorer economic status, lower education, people with family history of heart disease, smoking (especially unstable angina and myocardial infarction), positive troponin (especially in unstable angina and myocardial infarction), had a history of pulmonary and renal problems there were statistically significant more than others $(\mathrm{P}<0.05)$.

However, there was no significant relationship between cardiovascular disease with diabetes and family history of diabetes $(\mathrm{P}<$. $(0.05$

Also, there was a significant relationship between the mean systolic blood pressure, diastolic and blood glucose in patients and type of cardiovascular disease ( $\mathrm{P}$ $<0.05$ ). (Figure 2).

\section{Figure 1. Frequency of Types cardiovascular diseases by gender in patients with cardiac complications.}

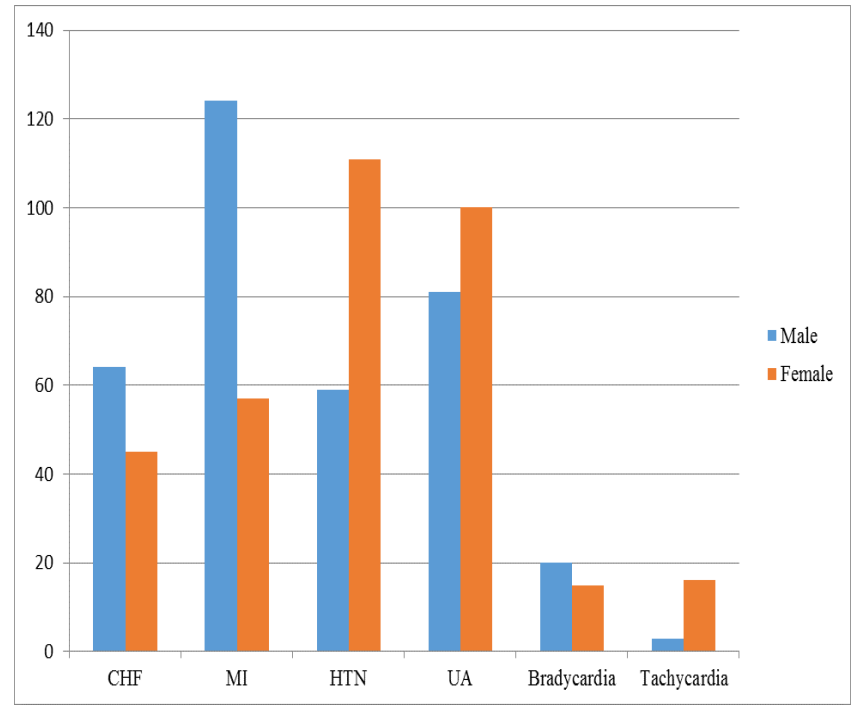


Figure 2. The average of blood pressure of systole, diastole and blood glucose in patients with cardiac complications.

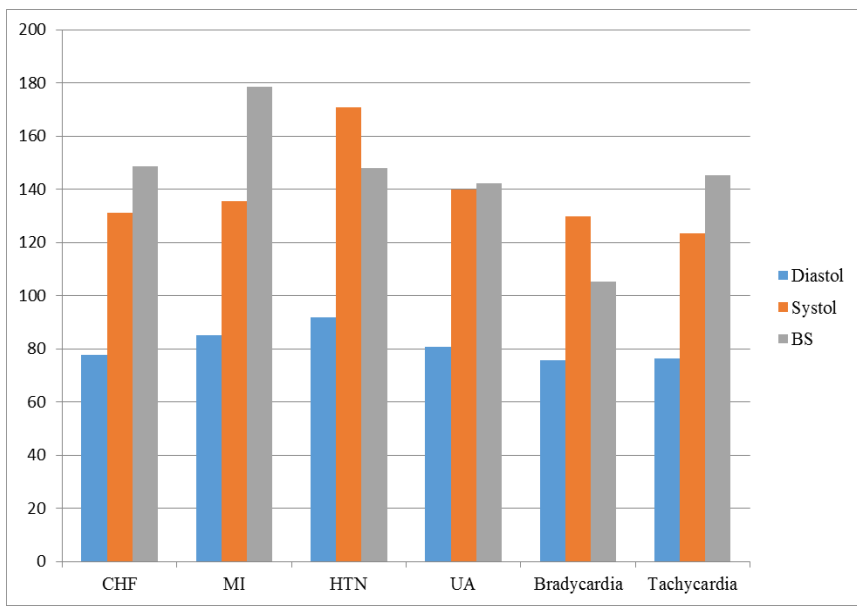

\section{Discussion}

Among the chronic diseases, cardiovascular disease is the most important and most common cause of death in many countries (1-3) In all countries, the risk factors for cardiovascular disease are associated with a significant health burden (4). The more people are exposed to more risk factors, the more likely they are to develop heart disease (15). By increasing the level of education, screening and controlling these factors, it can be prevented from developing cardiovascular disease or by delayed (16). Informing people about the role of risk factors in developing cardiovascular disease can be a way to combat these risk factors. The aim of this study was to determine Some risk factors and effective factors on cardiovascular diseases incidence by studying the cases of 705 patients referred to the Hospital of 22 Bahman in Masjed Soleyman that were residing in Masjed Soleyman, Lali and Andika.

In this study, 705 patients with cardiovascular disease were examined, that of these, $26.2 \%$ had diabetes, $18.4 \%$ had a family history of diabetes, $86.8 \%$ had a history of heart disease and $50.8 \%$ had a family history of heart disease. Troponin testing was positive in $30.6 \%$ of cases. $12.6 \%$ of patients had a history of smoking, 98.7 percent of patients used the drug, $2.3 \%$ had a history of vision problems, $12.8 \%$ had a history of kidney problems, $6.4 \%$ had a history of digestive problems. And Chi-square test showed a significant relationship between gender and type of cardiovascular disease $(\mathrm{P}<0.0001)$. This means that the prevalence of hypertension and unstable angina in women and the prevalence of congestive heart failure and myocardial infarction was higher in men. In a study quoted in a study (17), heart disease was associated with high blood pressure in men and diabetes in women, and there was a direct and significant relationship between ischemic changes with hypertension alone. The results of another study showed that $10.96 \%$ of type 2 diabetic patients were suffering from ischemic heart disease (18). Another study, quoted in the study (17), found a significant relationship between diabetes and ischemic changes in electrocardiography. But the results of another study, from 573 diabetic patients, showed that the lack of blood pressure, creatinine, and diastolic blood pressure at normal levels, despite high systolic blood pressure, did not increase the likelihood of myocardial infarction (19).
Other study results also showed that the prevalence of cardiovascular disease and hypertension in the elderly was 2.32 and $7.46 \%$, respectively. The results also showed that there is a significant difference between the use and non-use of cigarettes in relation to cardiovascular diseases and between the incidence and absence of hypertension in terms of cardiovascular disease. It also showed that the prevalence of cardiovascular diseases and high blood pressure in the elderly of the Isfahan retirement center is more than expected. The study also found that walking, table tennis, exercise, biking in the middle age had an impact on cardiovascular disease. Generally speaking, cardiovascular disease is one of the most important diseases of aging, including a progressive development that begins with childhood and manifests itself predominantly in adulthood from middle age. On the other hand, lack of movement increases all major causes of death and doubles the risk of cardiovascular disease. The WHO recommends that physical activity be at least 30 minutes a day, which can be one of the most important factors in the prevention of cardiovascular disease (20).

In this study, the mean blood glucose level of these patients was $151.55 \pm 87.62$. Also, there was a significant relationship between the mean systolic blood pressure, diastolic and blood glucose in patients and type of cardiovascular disease $(\mathrm{P}<0.05)$. In a study, they reported 4.6 and 4.5 milli-liter blood sugar, respectively, in men and women in Singapore. They also reported a positive and significant relationship between men's blood glucose (BMI, BF, WSR, WC and HC) (21). In another study, more than $28 \%$ of the subjects had a blood glucose greater than $150 \mathrm{mg} / \mathrm{dL}$ and about $62 \%$ had a high blood sugar level of $126 \mathrm{mg} / \mathrm{dL}$. In addition, the results of this study showed that the prevalence of cardiovascular disease was higher in a population with high blood glucose (22). Hassan Khani and colleagues also in a study that conducted to determine the prevalence of some cardiovascular risk factors and their relationship with gender in nurses, the rate of blood glucose was $11.5 \%$ in high level nurses (23). Also, in a study to investigate the prevalence of cardiovascular risk factors in taxi drivers in Yazd, the subjects expressed a glucose level of 101.62 $\mathrm{mg} / \mathrm{dL}$, that showed a high level (24).

In general, it can be said that diabetes, high blood pressure, high blood lipedema, smoking, lack of activity, high blood sugar and other causes that are a major risk factor for vascular disease, and by controlling them, can reduce the amount of these diseases and their costs.

\section{Conclusion}

The results of this study showed that cardiovascular disease is also found in people with a poorer economic status, lower education, people with family history of heart disease, smoking (especially unstable angina and myocardial infarction), positive troponin (especially in unstable angina and myocardial infarction), had a history of pulmonary and renal problems there were statistically significant more than others. Therefore, it is necessary to take necessary educational measures to reduce some of these risk factors. Also It is necessary to be done nutrition advice by nutritionists to reduce cardiovascular disease in these patients. 


\section{Acknowledgment}

The researchers in this study appreciated and thanked everyone who cooperated with us.

\section{Conflict of interest}

There are no conflicts of interest in this study.

\section{References}

1. Adavi A, Fathi Marghmalaki R, Madmoli Y, Fathi Marghmalaki R, Madmoli M. The Effect of Stress Management on Anxiety of Females with Hypertension. IJNR. 2016; 11 (5) :7-12

2. Adavi A, Marghmalaki F, Madmoli Y, Madmoli M. Investigate the effectiveness of cognitive-behavioral stress management intervention on aggression in women with hypertension. Journal of Clinical Nursing and Midwifery. 2017;4(3).

3. Madmoli M, Eilami O, Rezaie K, Aliabad MA, Moslemirad M. Diabetes and the risk of suffering cardiovascular Diseases: A two-year retrospective study. International Journal of Ecosystems and Ecology Science (IJEES). 2018 Jun;8(3): 649-56.

4. Davati A, Alikhah A, Safabakhsh M, Gharebaghi R, Razzaghi MH, Mehri M, et al. Effects of Education on Students' Parents' Knowledge. Med Sci J Islamic Azad Univ Tehran Med Branch. 2006;16(1):35-8.

5. Mashali H, Toleideh F, Rahmani R, Darabiyan P, Madmoli M. The predictive role of Hyperlipidemia in the incidence of ACS in patients referring to Shahidzadeh Hospital in Behbahan in 20162017. Medical Science, 2018, 22(94), 566-570

6. Sanaei Dashty A, Emami SR, Pazoki R, Ryahi F. the Knowledge of Cardiovascular Risk Factors in Elementary School Students in Bushehr Port the Persian Gulf Healthy Heart Project. Iran South Med J. 2005;8(1):68-74.

7. Grau M2. Bongard V, Fito M, Ruidavets JB, Sala J, Taraszkiewicz D. Prevalence of cardiovascular risk factors in men with stable coronary heart disease in France and Spain. Arch Cardiovasc Dis 2010; 103:8089.

8. World Health Organization 2006 Available at: http: // wwwwhoint/ncd surveillance infobase/web/ InfoBasePolicyMaker/reports/ReporterFullViewaspx? $\mathrm{id}=5$ About 4 pages Accessed June 15, 2007

9. Shafiei, Z., Babaee, S. \& Nazari, A. 2013. Relationship between Mood State and Quality of Life in Patients Undergoing Coronary Artery Bypass Graft Surgery. Iran Journal of Nursing, 26, 57-67.

10. Ai Ikeda, Hiroyasu.et al. (2007) Marital status and mortality among Japanese men and women: The Japan collaborative cohort study. BMC Public Health. 7:73.

11. Panthee B, Kritpracha C. Anxiety and Quality of life in patients with Myocardial infarction. Nurse Med $\mathrm{J}$ Nurs 2011;1(1): 105-15.

12. Park K \& Park J, Malek Afzalei H, et al. Darsnamh preventive and social medicine. Gap, 2005: 630-639. (Persian)
13. Mousavinasab N, Yazdani Cherat J, Bagheri B, Bakhti F, Bakhti Z. Identifying the Risk Factors for Cardiovascular Disease in Individuals Aged above 35 Years Using Logistic Regression Model. J Mazandaran Univ Med Sci. 2017; 26 (144) :50-56

14. Mohtasham Amiri Z. Study of the major risk factors for cardiovascular diseases in physicians of Guilan province in 2007. Scientific Journal of the Medical Council of the Islamic Republic of Iran. 2010; 28 (2): 184-189

15. Salehi, L., Taghdisi, M., Ghasemi, H. \& Shokervash, B. 2010. To Identify the Facilitator and Barrier Factors of Physical Activity among Elderly People in Tehran. Iranian Journal of Epidemiology, 6, 7-15.

16. Leon, A. S., Franklin, B. A., Costa, F. \& Balady, G. J. 2014. Cardiac Rehabilitation and Secondary Prevention of Coronary Heart Disease. Circulation, 369-376.

17. Fakhrzadeh H, Larijani B, Bandarian F, Adibi H, Samavat T, Malek Afzali H, Javadi HR, Hojatzadeh E. The relationship between ischemic heart disease and coronary risk factors in population aged over 25 in Qazvin: A population-based study. J Qazvin Univ Med Sci. 2005;35(9):26-34.

18. Rasi V, Arabi M. The relation between body mass index and prevalence of ischemic heart disease in type 2 diabetic patients. Razi Journal of Medical Sciences.39-34:(4)3;2019

19. Shariatpanahi SP, Habibi D, Rafiei M, Ghandi Y, Anvari M. Determination of Relations between Systolic Blood Pressure and Heart Attack in Patients with Type 2 Diabetes with Association Rules.20183; $.50-44:(8)$

20. Sharifirad G, Mohebbi S, Matlabi M. The relationship of physical activity in middle age and cardiovascular problems in old age in retired people in Isfahan, 2006. Horizon Med Sic. 2007; 13 (2) :5763

21. Bi X, Tey SL, Leong C, Quek R, Loo YT, Henry CJ. Correlation of adiposity indices with cardiovascular disease risk factors in healthy adults of Singapore: a cross-sectional study. BMC Obes 2016; 3:33.

22. Delpisheh A, Azizi H, Dantalab Esmaeili E, Haghiri L, Karimi G, Abbasi F. The qulity of care and blood suger control in type II diabetic patients of rural areae under the care by family physicians. Iran J Diabetes Metab 2016; 14:189-98.

23. Hasankhani H, Asadi P, Hasanlo M, Fakhri M, Zeinalzadeh AH, Rabie SS, et al. Frequency of the cardiovascular risk factors and their relationship with nurse's gender in intensive Care unit and emergency department, Kermanshah, Iran. Iran J Emerg Care 2017; 1:22-31.

24. Hachesu VR, Feli SN, Sakhvidi MJ. Prevalence of cardiovascular risk factors among taxi drivers in Yazd, Iran, 2016. J Commun Health Res 2017; 6:2006. 